\title{
Product Integration for Weakly Singular Integral Equations
}

\author{
By Claus Schneider
}

\begin{abstract}
The product integration method is used for the numerical solution of weakly singular integral equations of the second kind. These equations often have solutions which have derivative singularities at the endpoints of the range of integration. Therefore, the order of convergence results of de Hoog and Weiss for smooth solutions do not hold in general. In this paper it is shown that their results may be regained for the general case by using an appropriate nonuniform mesh. The spacing of the knot points is defined by the behavior of the solution at the endpoints. If the solution is smooth enough the mesh becomes uniform. Numerical examples are given.
\end{abstract}

In this paper we consider the numerical solution of the second kind equation

$$
\lambda x(s)-\int_{a}^{b} g_{\alpha}(|s-t|) k(s, t) x(t) d t=y(s), \quad s \in J:=[a, b],
$$

$$
\begin{aligned}
\text { with } g_{\alpha}(s) & :=s^{\alpha-1}, 0<\alpha<1, g_{1}(s):=\log (s), \\
\text { where } 0 & \neq \lambda \in \mathbf{R}, y \in C(J), \text { and } k \in C(J \times J) .
\end{aligned}
$$

Symbolically, we write (1) as $(\lambda I-K) x=y$. Then $K$ is a compact operator on $(C(J)$, sup), and a unique continuous solution of (1) exists, if and only if $\lambda$ is not an eigenvalue of $K$; cf. Atkinson [2].

Integral equations of this kind arise from potential problems, Dirichlet problems, the description of hydrodynamic interaction between elements of a polymer chain in solution, mathematical problems of radiative equilibrium, and transport problems; see Delves and Walsh [8], Atkinson [3], Auer and Gardner [4], [5], Hopf [9], Kaper and Kellogg [10].

Now let $S_{n, m}$ be the set of piecewise polynomials of degree $m-1$ on the grid $\Delta:=\left\{a=\tau_{0}<\tau_{1}<\cdots<\tau_{n}=b\right\},\left\{0 \leqslant s_{1}<s_{2}<\cdots<s_{m}<1\right\}$ a partition of the unit interval, and $P_{n}: C(J) \rightarrow S_{n, m}$ the interpolatory projection defined so that $P_{n} v$ interpolates $v \in C(J)$ at the $m$ points $t_{i j}:=\tau_{i}+s_{j}\left(\tau_{i+1}-\tau_{i}\right)$ on each subinterval $\left[\tau_{i}, \tau_{i+1}\right], i=O(1) n-1, j=1(1) m$. Then a product integration solution to (1) is a function $x_{n}$ satisfying

$$
\begin{array}{r}
\lambda x_{n}(s)-\int_{a}^{b} g_{\alpha}(|s-t|)\left[P_{n}\left(k(s,) x_{n}\right)\right](t) d t=y(s), \quad s \in J, \\
\text { or }\left(\lambda I-K_{n}\right) x_{n}=y, \text { respectively. }
\end{array}
$$

It can be shown that $K_{n}$ is a sequence of consistent, collectively compact operators approximating $K$; cf. Anselone [1], Atkinson [2]. Thus, it follows-if $\lambda$ is not an

Received February 14, 1980.

1980 Mathematics Subject Classification. Primary 45B05, 65R20.

(C) 1981 American Mathematical Society $0025-5718 / 81 / 0000-0016 / \$ 02.75$ 
eigenvalue of $K$-that for sufficiently large $n$ there exists a uniquely defined solution $x_{n}$ of $(2)$ and

$$
\left\|x_{n}-x\right\|_{\infty} \leqslant C\left\|K x-K_{n} x\right\|_{\infty}
$$

with $C$ depending only on $K$ and $\lambda$; see Anselone [1].

Assuming a smooth solution and a uniform grid $\Delta, \tau_{i}=a+(b-a) i / n, i=$ $0(1) n$, de Hoog and Weiss showed in [7] that

$$
\begin{array}{r}
M:=\int_{0}^{1} \prod_{j=1}^{m}\left(s_{j}-s\right) d s \neq 0 \text { and } x \in C^{m}(J) \text { imply }\left\|x-x_{n}\right\|_{\infty}=\theta\left(n^{-m}\right), \\
\text { if } M=0 \text { and } x \in C^{m+1}(J),
\end{array}
$$

then

$$
\left\|x-x_{n}\right\|_{\infty}= \begin{cases}\Theta\left(n^{-m-\alpha}\right), & 0<\alpha<1, \\ \vartheta\left(n^{-m-1} \log (n)\right), & \alpha=1 .\end{cases}
$$

I.e., a quadrature rule with $M=0$ (for example product Simpson or product Gauss) yields better convergence.

Unfortunately, weakly singular integral equations often have solutions containing mild singularities at the endpoints $a$ and $b$ introduced by the kernel. Then the best result for uniform grids-proven by Chandler [6]-is

$$
\left\|x-x_{n}\right\|_{\infty}= \begin{cases}\vartheta\left(n^{-2 \alpha}\right), & 0<\alpha<1, \\ \vartheta\left(n^{-2} \log (n)\right), & \alpha=1 .\end{cases}
$$

Nevertheless, we will show that the order of convergence result (4) remains true if an appropriate nonuniform mesh $\Delta$ is used. At first, let us summarize regularity properties of the solution $x$ (cf. [15]) in a form which will be sufficient for our purposes.

TheOrem 1. Let $\mu \in \mathbf{N}, k \in C^{\mu+1}(J \times J), y \in C^{\mu}(J), 0<\alpha<1, \lambda$ not an eigenvalue of $K$, and $x$ the solution of (1). Then $x \in C^{\mu}(\operatorname{int}(J))$, and there exists a $\beta \in[\alpha, \mu]$, if $0<\alpha<1$, or, if $\alpha=1$ (the logarithmic case) for any $\varepsilon \in(0,1)$ there exists $a \beta \in[1-\varepsilon, \mu]$ such that

$$
\left|x^{(i)}(s)\right| \leqslant C_{i} \begin{cases}(s-a)^{\beta-i}, & a<s \leqslant(a+b) / 2, \\ (b-s)^{\beta-i}, & (a+b) / 2 \leqslant s<b,\end{cases}
$$

with Hölder constants $C_{i}, i=1(1) \mu$. I.e., $x$ is of type $(\beta, \mu,\{a, b\})$, a notion introduced by Rice [12].

REMARKS. 1. In general, $\beta$ will not be greater than $\alpha$ or $1-\varepsilon$, respectively, and then $x \notin C^{1}(J)$.

2. The statements of the theorem remain valid if $y$ is only assumed to be of type $(\alpha, \mu,\{a, b\})$ or if $y$ is of type $(1-\varepsilon, \mu,\{a, b\})$ for any $\varepsilon \in(0,1)$.

3. A function of type $(\gamma, \mu,\{a, b\})$ with $N-1 \leqslant \gamma<N \in \mathbf{N}, N<\mu$, is an element of $C^{N-1}(J)$.

4. For other characterizations of the solution and further smoothness results see Richter [13], Kaper and Kellogg [10], Pitkäranta [11], Chandler [6]. 
In order to approximate such functions by piecewise polynomials Rice [12] introduced a mesh $\Delta$ with knot points

$$
\begin{aligned}
\tau_{i} & :=a+\left(\frac{b-a}{2}\right)\left(\frac{2 i}{n}\right)^{q}, \quad 0 \leqslant i \leqslant n / 2, \\
\tau_{i} & :=(a+b)-\tau_{n-i}, \quad n / 2 \leqslant i \leqslant n .
\end{aligned}
$$

A first order of convergence result is obtained with this mesh.

THEOREM 2. If $x$ is the solution of (1), $m \geqslant \beta \geqslant \alpha, x$ of type $(\beta, m,\{a, b\})$ or $x$ of type $(\beta-\varepsilon, m,\{a, b\})$ for any $\varepsilon \in(0, \beta)$-i.e. $x$ contains logarithmic endpoint singularities-, and $x_{n}$ the solution of (2) with the nonuniform mesh (5) and the grading exponent $q:=m / \beta$ or $q:=m /(\beta-\varepsilon)$ in the logarithmic case, $0<\varepsilon<\beta$, then

$$
\left\|x-x_{n}\right\|_{\infty}=\mathcal{O}\left(n^{-m}\right) \text {. }
$$

Proof. By (3),

$$
\left\|x-x_{n}\right\|_{\infty} \leqslant C\left\|K x-K_{n} x\right\|_{\infty} \leqslant C^{\prime} \max _{s \in J}\left\|k(s,) x-P_{n}[k(s,) x]\right\|_{\infty}
$$

and, independently of $s$, the error in the approximation of $k(s)$,$x by P_{n}$ is of order $n^{-m}$ if $q$ is chosen as supposed; cf. Rice [12] and [14].

Thus, the result (4) of de Hoog and Weiss is regained in the case $M \neq 0$, and a smooth $x\left(x \in C^{m}(J)\right.$, i.e. $\left.\beta=m\right)$ leads to the uniform mesh. Now we will study the influence of the partition $\left\{s_{j}\right\}$ which characterizes the quadrature rule used in product integration.

TheOREM 3. Let $M=0$. If $x$ is the solution of (1), $m+1 \geqslant \beta \geqslant \alpha, x$ of type $(\beta, m+1,\{a, b\})$ or $x$ of type $(\beta-\varepsilon, m+1,\{a, b\})$ for any $\varepsilon \in(0, \beta), x_{n}$ the solution of (2) with the nonuniform mesh (5) and the grading exponent $q:=$ $(\alpha+m+1) /(\alpha+\beta)$ or $q:=(\alpha+m+1) /(\alpha+\beta-\varepsilon)$ in the logarithmic case, $0<\varepsilon<\beta$, then

$$
\left\|x-x_{n}\right\|_{\infty}= \begin{cases}\Theta\left(n^{-m-\alpha}\right), & 0<\alpha<1 \\ \vartheta\left(n^{-m-1} \log (n)\right), & \alpha=1 .\end{cases}
$$

Proof. The proof will show that there is no loss of generality by taking $k(s, t) \equiv$ 1.

Let

$$
E\left(g_{\alpha}, x\right):=\int_{a}^{b} g_{\alpha}(|s-t|)\left\{x(t)-\left(P_{n} x\right)(t)\right\} d t
$$

be the error committed by the product integration. Further, define

$$
\begin{aligned}
E_{i}\left(g_{\alpha}, x\right) & :=\int_{I_{i}} g_{\alpha}(|s-t|)\left\{\left(I-P_{n}\right) x\right\}(t) d t \quad \text { and } \\
I_{i} & :=\left[\tau_{i}, \tau_{i+1}\right], \quad i=0(1) n-1 .
\end{aligned}
$$


If $\left.\phi\right|_{I_{i}}=$ const $=: \phi_{i}$ is a piecewise constant function, then

$$
\begin{aligned}
\left|E_{i}\left(g_{\alpha}, x\right)\right| & =\left|E_{i}\left(g_{\alpha}-\phi, x\right)+E_{i}(\phi, x)\right| \\
& \leqslant\left\|g_{\alpha}-\phi\right\|_{1, I_{i}}\left\|x-P_{n} x\right\|_{\infty, I_{i}}+\left|\phi_{i}\right|\left|\int_{I_{i}}\left(x-P_{n} x\right)(t) d t\right| .
\end{aligned}
$$

Now let

$$
\phi_{i}:=\frac{1}{\tau_{i+1}-\tau_{i}} \int_{I_{i}} g_{\alpha}(|s-t|) d t
$$

Then Chandler [6] showed:

(i) $\quad \gamma<\alpha \Rightarrow \sum_{i=0}^{n-1}\left(\tau_{i+1}-\tau_{i}\right)^{-\gamma}\left\|g_{\alpha}-\phi\right\|_{1, I_{i}}<C \begin{cases}n^{-\alpha+\gamma}, & 0<\alpha<1, \\ n^{-1+\gamma} \log (n), & \alpha=1 .\end{cases}$

(ii)

$$
0<\alpha<1: \sup _{i=0}^{n-1}\left(\tau_{i+1}-\tau_{i}\right)^{1-\alpha}\left|\phi_{i}\right|<C,
$$

$$
\alpha=1: \sup _{i=0}^{n-1} \frac{\left|\phi_{i}\right|}{\left|\log \left(\tau_{i+1}-\tau_{i}\right)\right|}<C
$$

and the constants $C$ are independent of $n$ and $s$.

Summing the inequality of (6) over $i$ shows that

$$
\begin{aligned}
\left|E\left(g_{\alpha}, x\right)\right| \leqslant & \sum_{i=0}^{n-1}\left\|g_{\alpha}-\phi\right\|_{1, I_{i}}\left\|x-P_{n} x\right\|_{\infty, I_{i}} \\
& +\sup _{i=0}^{n-1}\left(\tau_{i+1}-\tau_{i}\right)^{1-\alpha}\left|\phi_{i}\right| \sum_{i=0}^{n-1}\left(\tau_{i+1}-\tau_{i}\right)^{\alpha-1}\left|\int_{I_{i}}\left(x-P_{n} x\right)(t) d t\right|
\end{aligned}
$$

Let us first study the last term in (7): if $i=0$ or $i=n-1$, then

$$
\left|\int_{I_{i}}\left(x-P_{n} x\right)(t) d t\right| \leqslant C\left(\tau_{1}-\tau_{0}\right)^{\beta+1}=C^{\prime} n^{-q(\beta+1)}
$$

cf. Rice [12]. In the other intervals the error of the quadrature rule $(M=0)$ may be estimated by

$$
\begin{aligned}
\left|\int_{I_{i}}\left(x-P_{n} x\right)(t) d t\right| & <C\left(\tau_{i+1}-\tau_{i}\right)^{m+2}\left\|x^{(m+1)}\right\|_{\infty, I_{i}} \\
& \leqslant C^{\prime}\left[\left(\frac{j+1}{n}\right)^{q}-\left(\frac{j}{n}\right)^{q}\right]^{m+2}\left(\frac{j}{n}\right)^{q(\beta-m-1)}
\end{aligned}
$$

with

$$
j:= \begin{cases}i, & 0<i<n / 2 \\ n-i-1, & n / 2<i<n-1,\end{cases}
$$


because $x$ is of type $(\beta, m+1,\{a, b\})$; cf. Rice [12]. Now (ii) implies

$$
\begin{aligned}
|E(\phi, x)|= & \sum_{i=0}^{n-1}\left|E_{i}(\phi, x)\right| \\
\leqslant & C\left\{\left(\tau_{1}-\tau_{0}\right)^{\alpha-1} n^{-q(\beta+1)}\right. \\
& \left.\quad+\sum_{i=1}^{n / 2-1}\left(\tau_{i+1}-\tau_{i}\right)^{\alpha-1}\left[\left(\frac{i+1}{n}\right)^{q}-\left(\frac{i}{n}\right)^{q}\right]^{m+2}\left(\frac{i}{n}\right)^{q(\beta-m-1)}\right\} \\
\leqslant & C^{\prime} n^{-q(\alpha+\beta)}\left\{1+\sum_{i=1}^{n / 2-1} i^{(q-1)(\alpha-1+m+2)+q(\beta-m-1)}\right\} \\
= & C^{\prime} n^{-q(\alpha+\beta)}\left\{1+\sum_{i=1}^{n / 2-1} i^{q(\alpha+\beta)-(\alpha+m+1)}\right\} \\
= & C^{\prime} n^{-q(\alpha+\beta)} n / 2=\mathcal{O}\left(n^{-m-\alpha}\right) .
\end{aligned}
$$

Let us now inspect the first term of (7), and we will further assume that $\alpha<\beta<$ $m$ :

$$
\begin{aligned}
\left|E\left(g_{\alpha}-\phi, x\right)\right| \leqslant C\left\{\left\|g_{\alpha}-\phi\right\|_{1, I_{0}}\left(\tau_{1}-\tau_{0}\right)^{\beta}+\left\|g_{\alpha}-\phi\right\|_{1, I_{n-1}}\left(\tau_{n}-\tau_{n-1}\right)^{\beta}\right. & \\
& \left.+\sum_{i=1}^{n-2}\left\|g_{\alpha}-\phi\right\|_{1, I_{i}}\left(\tau_{i+1}-\tau_{i}\right)^{m}\left\|x^{(m)}\right\|_{\infty, I_{i}}\right\}
\end{aligned}
$$

(cf. Rice [12] and [14])

$$
\begin{aligned}
\leqslant C^{\prime}\left\{\left\|g_{\alpha}-\phi\right\|_{1, I_{0}}\left(\tau_{1}-a\right)^{\beta}+\left\|g_{\alpha}-\phi\right\|_{1, I_{n-1}}\left(b-\tau_{n-1}\right)^{\beta}\right. \\
\left.+\sum_{i=1}^{n-2}\left\|g_{\alpha}-\phi\right\|_{1, I_{i}}\left(\tau_{i+1}-\tau_{i}\right)^{m}\left(\frac{j}{n}\right)^{q(\beta-m)}\right\}
\end{aligned}
$$

with

$$
\begin{aligned}
& j: \begin{cases}1, & i=0 \text { or } i=n-1, \\
i, & 0<i<n / 2, \\
n-i-1, & n / 2 \leqslant i<n-1,\end{cases} \\
&<C^{\prime \prime}\left\{n^{-q(\beta+\gamma)}\left[\left\|g_{\alpha}-\phi\right\|_{1, I_{0}}\left(\tau_{1}-a\right)^{-\gamma}+\left\|g_{\alpha}-\phi\right\|_{1, I_{n-1}}\left(b-\tau_{n-1}\right)^{-\gamma}\right]\right. \\
&\left.\quad+\sum_{i=1}^{n-2}\left\|g_{\alpha}-\phi\right\|_{1, I_{i}}\left(\tau_{i+1}-\tau_{i}\right)^{-\gamma} \frac{j^{(q-1)(m+\gamma)}}{n^{q(m+\gamma)}}\left(\frac{j}{n}\right)^{q(\beta-m)}\right\} \\
&=C^{\prime \prime} n^{-q(\beta+\gamma)} \sum_{i=0}^{n-1}\left\|g_{\alpha}-\phi\right\|_{1, I_{i}}\left(\tau_{i+1}-\tau_{i}\right)^{-\gamma} j^{q(\beta+\gamma)-(m+\gamma)} .
\end{aligned}
$$

If $q:=(m+\gamma) /(\beta+\gamma)$, then this expression is equal to

$$
=C^{\prime \prime} n^{-m-\gamma} \sum_{i=0}^{n-1}\left\|g_{\alpha}-\phi\right\|_{1, I_{i}}\left(\tau_{i+1}-\tau_{i}\right)^{-\gamma} \text {. }
$$

If $\gamma<\alpha$, then (i) implies

$$
\leqslant C^{\prime \prime \prime} n^{-m-\gamma} n^{-\alpha+\gamma}=\theta\left(n^{-m-\alpha}\right) .
$$


On the other hand, we have already chosen the grading exponent $q=$ $(m+\alpha+1) /(\alpha+\beta)$. Therefore taking $\gamma=\alpha(m-\beta-\beta / \alpha) /(m-\beta+1)$, this $\gamma$ is well defined and smaller than $\alpha$ for $0<\alpha \leqslant \beta<m$. If $m+1 \geqslant \beta \geqslant m$, then $x \in C^{m}(J)$ and

$$
\left|E\left(g_{\alpha}-\phi, x\right)\right| \leqslant C \sum_{i=0}^{n-1}\left\|g_{\alpha}-\phi\right\|_{1, l_{i}}\left(\tau_{i+1}-\tau_{i}\right)^{m}=\theta\left(n^{-\alpha-m}\right)
$$

by (i) for any $q>0$. The modifications for the logarithmic case are rather technical and therefore omitted.

By the theorems, the order of convergence result (4) is now established for the product integration solution of a weakly singular integral equation (1) and again a smooth $x\left(x \in C^{m+1}(J)\right.$; i.e., $\left.\beta=m+1\right)$ yields the uniform mesh. The grading exponent in the last two theorems is optimal in the sense that a $q$ smaller than supposed leads to a smaller order of convergence. If there is no knowledge in advance that $\beta$ happens to be greater than $\alpha$ (i.e., $x$ is smoother than expected), and if for this reason the grading exponent used is greater than necessary, then the order of convergence does not decrease.

For numerical verification of the above theorems we consider two examples. (All computations were done on the HB 66/80 of the Rechenzentrum der Johannes Gutenberg-Universität Mainz, in double precision.)

Example 1. $a=0, b=1, \alpha=0.5, \lambda=2, k(s, t)=1$, and $y$ is chosen so that $x(s)=2(\sqrt{s}+\sqrt{1-s})$. Then $x$ is of type $(0.5, j,\{0,1\})$ for any $j \in \mathbf{N}$; i.e., $\beta=\alpha=0.5$, but $y$ is of type $(1-\varepsilon, j,\{0,1\})$ for any $\varepsilon \in(0,1)$ and $j \in \mathbf{N}$.

$$
\max _{i, j}\left|x\left(t_{i j}\right)-x_{n}\left(t_{i j}\right)\right|, \quad i=0(1) n-1, \quad j=1(1) m:
$$

\begin{tabular}{c|c|c|c|c|c|c} 
& \multicolumn{2}{|c|}{$\begin{array}{c}\text { Trapezoidal rule } \\
m=2, M \neq 0\end{array}$} & \multicolumn{2}{c|}{$\begin{array}{c}\text { Simpson's rule } \\
m=3, M=0\end{array}$} & \multicolumn{2}{c}{$\begin{array}{c}\text { Gauss-Lobatto } \\
m=4, M=0\end{array}$} \\
\hline$n(m-1)$ & $q=\frac{m}{\beta}$ & $q=1$ & $q=\frac{m+\alpha+1}{\alpha+\beta}$ & $q=1$ & $q=\frac{m+\alpha+1}{\alpha+\beta}$ & $q=1$ \\
& & & & & \\
\hline 24 & $1.63_{10}-2$ & $2.65_{10}-2$ & $1.17_{10}-3$ & $9.70_{10}-3$ & $6.91_{10}-4$ & $6.27_{10}-3$ \\
48 & $4.15_{10}-3$ & $9.41_{10}-3$ & $8.58_{10}-5$ & $3.47_{10}-3$ & $2.36_{10}-5$ & $2.54_{10}-3$ \\
96 & $1.06_{10}-3$ & $3.33_{10}-3$ & $5.74_{10}-6$ & $1.81_{10}-3$ & $7.75_{10}-7$ & $1.43_{10}-3$ \\
192 & $2.68_{10}-4$ & $1.17_{10}-3$ & $3.71_{10}-7$ & $9.92_{10}-4$ & $4.10_{10}-8$ & $7.70_{10}-4$
\end{tabular}

Example 2. $a=0, b=1, \alpha=0.5, \lambda=2 \sqrt{2}, k(s, t)=1$, and $y$ is chosen so that $x(s)=2 \sqrt{2}[s(1-s)]^{3 / 4}$. Then $x$ and $y$ are of type $(3 / 4, j,\{0,1\})$ for any $j \in \mathbf{N}$; i.e., $\beta=3 / 4>\alpha$.

$$
\max _{i, j}\left|x\left(t_{i j}\right)-x_{n}\left(t_{i j}\right)\right|:
$$

\begin{tabular}{c|c|c|c|c|c|c} 
& \multicolumn{2}{|c|}{$\begin{array}{c}\text { Trapezoidal rule } \\
m=2, M \neq 0\end{array}$} & \multicolumn{2}{c|}{$\begin{array}{c}\text { Simpson's rule } \\
m=3, M=0\end{array}$} & \multicolumn{2}{c}{$\begin{array}{c}\text { Gauss-Lobatto } \\
m=4, M=0\end{array}$} \\
\hline$n(m-1)$ & $q=\frac{m}{\beta}$ & $q=1$ & $q=\frac{m+\alpha+1}{\alpha+\beta}$ & $q=1$ & $q=\frac{m+\alpha+1}{\alpha+\beta}$ & $q=1$ \\
\hline 24 & $8.56_{10}-3$ & $3.35_{10}-2$ & $5.91_{10}-4$ & $1.28_{10}-2$ & $1.77_{10}-3$ & $7.56_{10}-3$ \\
48 & $2.17_{10}-3$ & $1.44_{10}-2$ & $4.25_{10}-5$ & $4.43_{10}-3$ & $4.68_{10}-5$ & $2.90_{10}-3$ \\
96 & $5.77_{10}-4$ & $5.09_{10}-3$ & $2.94_{10}-6$ & $1.67_{10}-3$ & $1.38_{10}-6$ & $1.11_{10}-3$ \\
192 & $1.51_{10}-4$ & $1.64_{10}-3$ & $2.03_{10}-7$ & $6.42_{10}-4$ & $7.16_{10}-8$ & $4.26_{10}-4$
\end{tabular}


In either case, the nonuniform mesh yields better results, and the additional computational labor ( $n / 2$ exponentiations) is minimal.

Fachbereich Mathematik

Johannes Gutenberg-Universität

Saarstrasse 21

D6500 Mainz, West Germany

1. P. M. Anselone, Collectively Compact Operator Approximation Theory and Applications to Integral Equations, Prentice-Hall, Englewood Cliffs, N. J., 1971.

2. K. AtKInSON, "The numerical solution of Fredholm integral equations of the second kind," SIAM J. Numer. Anal., v. 4, 1967, pp. 337-348.

3. K. Atxinson, "The numerical solution of Fredholm integral equations of the second kind with singular kernels," Numer. Math., v. 19, 1972, pp. 248-259.

4. P. L. Auer \& C. S. GARdner, "Note on singular integral equations of the Kirkwood-Riseman type," J. Chem. Phys., v. 23, 1955, pp. 1545-1546.

5. P. L. Autr \& C. S. Gardner, "Solution of the Kirkwood-Riseman integral equation in the asymptotic limit," J. Chem. Phys., v. 23, 1955, pp. 1546-1547.

6. G. A. Chandere, Superconvergence of Numerical Solutions to Second Kind Integral Equations, Thesis, Australian National University, Canberra, 1979.

7. F. DE Hoog \& R. WeIss, “Asymptotic expansions for product integration,” Math. Comp., v. 27, 1973, pp. 295-306.

8. L. M. Delves \& J. Walsh (Editors), Numerical Solution of Integral Equations, Clarendon Press, Oxford, 1974.

9. E. Hopf, Mathematical Problems of Radiative Equilibrium, Stechert-Hafner Service Agency, New York, 1964.

10. H. G. KAPER \& R. B. KellogG, "Asymptotic behavior of the solution of the integral transport equation in slab geometry," SIAM J. Appl. Math., v. 32, 1977, pp. 191-200.

11. J. Pitkäranta, "On the differential properties of solutions to Fredholm equations with weakly singular kernels,” J. Inst. Math. Appl., v. 24, 1979, pp. 109-119.

12. J. R. RICE, "On the degree of convergence of nonlinear spline approximation," in Approximations with Special Emphasis on Spline Functions (I. J. Schoenberg, Ed.), Academic Press, New York, 1969, pp. 349-365.

13. G. R. RichteR, “On weakly singular Fredholm integral equations with displacement kernels," $J$. Math. Anal. Appl., v. 55, 1976, pp. 32-42.

14. C. SCHNEIDER, Beiträge zur numerischen Behandlung schwachsingulärer Fredholmscher Integralgleichungen zweiter Art, Thesis, Johannes Gutenberg-Universität, Mainz, 1977.

15. C. SCHNEIDER, "Regularity of the solution to a class of weakly singular Fredholm integral equations of the second kind," Integral Equations Operator Theory, v. 2, 1979, pp. 62-68. 